\title{
Esquizofrenia e direção veicular: uma revisão sistemática da literatura
}

\author{
Schizophrenia and vehicle driving: a systematic literature review \\ Carolina Fernandes ${ }^{1}$, Caio Del Arco Esper ${ }^{2}$, Carla Júlia Segre Faiman ${ }^{3}$
}

DOI: http://dx.doi.org/10.11606/issn.2317-2770.v22i2p72-80

\begin{abstract}
Fernandes C, Esper CDA, Faiman CJS. Esquizofrenia e direção veicular: uma revisão sistemática da literatura.
RESUMO: Introdução: O diagnóstico de esquizofrenia vem, ao longo dos anos, carregado de um processo histórico de transformações sob o olhar da sociedade. Nesse âmbito, a esquizofrenia é vista atualmente como doença degenerativa, de caráter demencial progressivo, com prejuízo global de suas funções e declínio cognitivo, gerando muito comumente, uma vida marcada por preconceito e exclusão, inclusive no âmbito de mobilidade no que se diz respeito à direção veicular. Concomitante a esses dados, existe uma escassez de trabalhos de investigação sobre acidentes de automóveis em condutores portadores de esquizofrenia, apesar de alguns estudos apontarem no sentido de maior potencial fator de risco. Objetivo: O objetivo desta revisão bibliográfica foi levantar estudos na literatura que tragam informações acerca do paciente portador de esquizofrenia e direção veicular, buscando responder se podem ou não ser liberados através do exame mental/psicológico. Método: Realizou-se uma revisão sistemática da literatura científica sobre a relação entre esquizofrenia e direção veicular. Resultados: Existem estudos que sugerem um maior número de infrações às regras de trânsito e acidentes de viação em pacientes esquizofrênicos, porém esta diferença não fora observada por outros autores. Houve uma tendência de distorção de feedback visual nos pacientes em relação à população geral, além de erros de processamento sensorial. Esquizofrênicos que dirigiam apresentaram-se melhores no exame do estado mental que esquizofrênicos não motoristas. As taxas de acidente encontradas foram quase idênticas nos grupos de pacientes com transtornos psicóticos e controle, e que além disso, taxas de acidentes com lesões podem ser equiparadas com as da população geral. Conclusão: Diante dos dados expostos, fica evidente a relação entre esquizofrenia e prejuízo cognitivo. Porém os estudos traçados não permitiram exercer com solidez a aferição destas perdas mentais, e tampouco demonstrar o impacto na direção veicular. Em suma, torna-se de extrema importância investigação mais apurada destes pacientes, para que não sejam indevidamente impedidos de exercer o ato da direção veicular. À medida que mais estudos surgirem, os profissionais da Medicina de Tráfego obterão mais embasamento teórico em sua avaliação mental.
\end{abstract}

PALAVRAS-CHAVE: Esquizofrenia; Condução de Veículo.

\footnotetext{
Coordenadora de Psiquiatria do Pronto Socorro Municipal da Lapa pela Associação Saúde da Família. Supervisora de Psiquiatria do município de Santana de Parnaíba.

2. Faculdade Estadual de Medicina de Marília.

3. Departamento de Medicina Legal, Ética Médica e Medicina Social e do Trabalho da Faculdade de Medicina da Universidade de São Paulo.

Endereço para correspondência: Carolina Fernandes, e-mail: carolinapsiq@gmail.com
} 


\section{INTRODUÇÃO}

$\mathbf{O}$ diagnóstico de esquizofrenia vem, ao longo dos anos, carregado de um processo histórico de transformações sob o olhar da sociedade. Sua descrição foi introduzida quando Emil Kraepelin, no final do século XIX, teve a percepção de que alguns pacientes apresentavam síndrome com manifestações comuns, semelhantes a um quadro demencial, e denominou esta entidade de "demência precoce”. Em seguida, Bleuler (1857-1939), apresentou o termo "esquizofrenia" (esquizo= divisão, phrenia= mente), e através de sintomas específicos como alucinações, delírios, frouxidão de associações, ambivalência, autismo e alterações do afeto, deu abertura para muitos outros percorrerem o caminho obscuro dotado de tantas dúvidas, principalmente no que se dizia respeito às suas causas, prognóstico e tratamento ${ }^{1}$.

Reunindo resultados de muitos estudos científicos, o DSM-V foi descrito e delineado de modo a visualizar as grandes questões humanas com abordagem dimensional em detrimento da classificatória, aplicando à esquizofrenia uma visão de um amplo espectro. Neste sentido, incluemse neste transtorno aqueles com alterações em um ou mais dos domínios a seguir: delírios, alucinações, pensamento desorganizado, comportamento motor grosseiramente desorganizado ou anormal (incluindo catatonia) e sintomas negativos, como anedonia, apatia, isolamento social, embotamento afetivo. Os subtipos antes apresentados (paranoide, hebefrênico, residual, indiferente, catatônico) foram eliminados nesta nova versão do DSM, pois há baixa limitação no diagnóstico, com baixa confiabilidade e sem diferenças significativas na resposta ao tratamento ou mudança no curso do tratamento ${ }^{2}$.

Exatamente por tratar-se de um quadro tão heterogêneo, pode-se encontrar pacientes em graus variados de acometimento funcional, como, por exemplo, no trabalho, nas relações interpessoais, autocuidado e na área acadêmica. Nesse âmbito, a esquizofrenia é vista atualmente como doença degenerativa, de caráter demencial progressivo, com prejuízo global das funções e declínio cognitivo, gerando, muito comumente, uma vida marcada por preconceito e exclusão ${ }^{3}$.

Sabe-se que fatores que contribuem para tal mau prognóstico são idade de início precoce, diversas hospitalizações, prevalência de sintomas negativos, baixo nível social ${ }^{4}$

Parece que a prevalência da esquizofrenia é dada entre 0,3 a $1 \%$, podendo variar entre países. A idade de pico do início no sexo masculino encontra-se entre 18-25 anos e nas mulheres entre $25-35$ anos, sendo mais raro início antes da puberdade e após os 50 anos de idade 5 .

Diante disso, constata-se que a esquizofrenia traz um potencial declínio da cognição, alterando em maior ou menor grau as instâncias psicopatológicas, principalmente a atenção voluntária e espontânea, a psicomotricidade, a sensopercepção e a crítica da realidade. Sendo doença crônica e progressiva, acomete as pessoas geralmente a partir da juventude, época em que representariam o maquinário produtivo da economia social, e que, muitas vezes, são privadas de suas atividades e interesses próprios de sua geração, como por exemplo, cursar uma faculdade, sociabilizar em festas, ter uma carreira laboral, ter independência de dirigir veículos.

Esses portadores do espectro da esquizofrenia podem apresentar alterações cognitivas e comportamentais, em variados graus, a depender da natureza da doença, de suas características, anos de duração, gravidade de sintomas, uso de psicofármacos, comorbidades clínicas e psiquiátricas, ambiente social a que pertencem. Estas variáveis tendem a comprometer, de maneira permanente, o seu desempenho na condução veicular ${ }^{6}$.

Nesta ação de conduzir um veículo, necessitase da utilização da percepção de estímulos externos, interpretação das situações, decisões rápidas, planejamento e execução, velocidade de processamento de feedback, atenção, domínio visuo-perceptivo-espacial, memória de trabalho, dentre outros?.

Concomitante a esses dados, existe uma escassez de trabalhos de investigação sobre acidentes de automóveis em condutores portadores de esquizofrenia, apesar de alguns estudos apontarem no sentido de maior potencial fator de risco ${ }^{8}$

Além do próprio transtorno ser composto por quadro clínico significativamente heterogêneo, o que pode por si só dificultar a padronização do risco apresentado no volante automotivo, existem outras variáveis que aumentam a complexidade deste universo clínico. Importante recordar que o consumo de psicotrópicos, principalmente no que diz respeito ao uso regular e prolongado, pode prejudicar secundariamente a condução veicular. Fica extremamente difícil diferenciar o impacto da doença dos efeitos dos fármacos ${ }^{9}$.

Também existem diferenças clínicas, e, portanto, cognitivas, entre os momentos de crise na esquizofrenia e o período de estabilidade. Alguns pacientes recuperamse quase que totalmente, e fora do surto retomam suas atividades rotineiras, com retorno de suas capacidades. Já, outros, carregam esses prejuízos por períodos persistentes ${ }^{10}$.

Entretanto, alguns estudos sugerem que há maior número de acidentes automobilísticos e infrações de trânsito envolvendo pacientes esquizofrênicos, em detrimento da população não psiquiátrica ${ }^{11,12}$.

Nesse sentido, coloca-se a avaliação psicológica como essencial, pois esta verifica os processos cognitivos, além do comportamento do candidato, através da análise dos traços de personalidade, controle emocional, impulsividade, autoconfiança, tolerância a frustrações, memórias auditiva e visual, controle de agressividade, relacionamento interpessoal, fluência do pensamento, sinais fóbicos, dentre outros ${ }^{13}$. 
Porém, segundo a mais recente Resolução do CONTRAN no 425 (Conselho Nacional de Trânsito), a avaliação psicológica é exigida apenas para fins de obtenção da permissão para dirigir, adição e/ou mudança de categoria e ocasião da renovação da carteira nacional de habilitação para o condutor que exercer atividade remunerada ao veículo ${ }^{14}$.

Neste contexto, somente a avaliação médica por não especialista, empiricamente, tem se mostrado como pouco sensível e específica para o exame mental, cujas técnicas não têm sido conclusivas na questão da capacidade de condução, talvez pela falta de domínio e formação na área específica.

Em muitas situações, pede-se ajuda recorrendo-se a laudos de especialistas e/ou juntas médicas e psicológicas na resposta destas questões, porém a quantidade de pedidos dessespareceresésignificativamentemenorem comparação com a proporção de pessoas com transtornos psiquiátricos, denotando que um grande número de pacientes não está sendo identificado pelos examinadores ${ }^{15}$.

Mesmo que o ato de dirigir seja considerado algo complexo, multifatorial, que exige habilidades cognitivas e experiência por parte do condutor, o fato é que cerca de $43 \%$ de portadores de esquizofrenia de meia-idade em diante, possuem carteira de habilitação e dirigem ativamente ${ }^{16}$.

A permissão para dirigir traz um importante papel da mobilidade coletiva, no que diz respeito à saúde, bemestar, participação na comunidade, redes sociais, trabalho, fonte de renda; inserindo o homem na sociedade ${ }^{17}$.

Dessa maneira, é preciso ter um olhar mais aprofundado para os pacientes esquizofrênicos durante suas avaliações mentais/psicológicas, para que seja viabilizada a possibilidade da condução veicular, e assim melhorar sua inclusão social, porém de forma embasada e segura, minimizando os riscos para si próprios e outros envolvidos no trânsito.

O objetivo desta revisão bibliográfica foi levantar estudos na literatura que tragam informações acerca do paciente portador de esquizofrenia e direção veicular, buscando responder se eles podem ou não ser liberados através do exame mental/psicológico. Busca-se trazer contribuições para o exame de aptidão física e mental para condutores e candidatos a condutores de veículos automotores.

\section{MÉTODO}

Realizou-se uma revisão sistemática da literatura científica sobre a relação entre esquizofrenia e direção veicular. A presente revisão foi baseada em pesquisa nas seguintes bases de dados científicas: PubMed; BVS (Biblioteca Virtual em Saúde) e Scielo (Scientific Eletronic Library Online). Não houve restrição quanto ao período da publicação.

Foram utilizados os seguintes descritores de assunto: automobile driving; schizophrenia, de acordo com os termos do Mesh (Medical Subject Headings).

Os critérios de seleção dos estudos incluíram: 1. Estudos originais e empíricos. 2. Estudos que estavam em periódicos científicos. 3.Estudos nos idiomas inglês, português e espanhol.

Foram estabelecidos os seguintes critérios de exclusão: 1. Estudos que davam maior ênfase ao efeito secundário de psicotrópicos e substâncias psicoativas. 2. Pesquisas que se distanciavam do transtorno psiquiátrico elegido (esquizofrenia).

Não houve procura por estudos não publicados. Somente um dos autores realizou o julgamento dos artigos a serem incluídos/excluídos, sua leitura e análise de dados.

\section{Estratégia de Busca e Seleção dos Artigos}

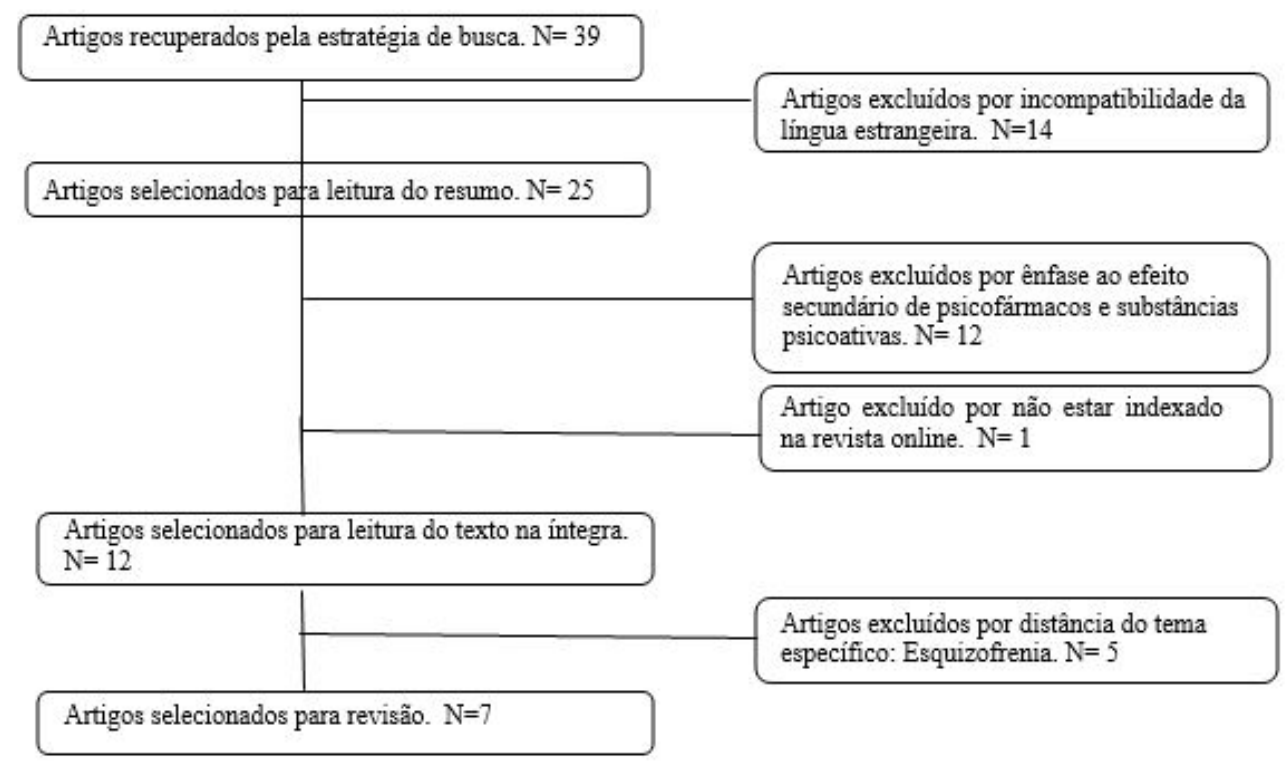




\section{RESULTADOS}

\section{Metodologia de aferição dos trabalhos levantados}

Dos artigos encontrados, a maior parte era de estudos observacionais (6), sendo 1 de revisão e outros 5 transversais. Apenas 1 estudo se propôs a realizar medida experimental. Sendo assim, na maioria dos estudos tornase impossível a determinação da prova causal, mas há boa fonte de hipóteses.

Além disso, trazem amostras com $\mathrm{N}$ variável, nem sempre refletindo a população real.

\section{Objetivos das pesquisas da literatura}

Em sua totalidade, tentaram estabelecer relação entre esquizofrenia e capacidade/performance na direção veicular. Uma vez encontrada alguma correlação, tentouse analisar alguma natureza encontrada.

Houve estudos que aprofundaram na questão do status funcional, explorando outros domínios como atividades laborais, independência pessoal, mobilidade individual $^{16,18}$

Dois outros artigos trouxeram como objetivo principal a avaliação específica de algumas atribuições mentais envolvidas no ato de dirigir, como, por exemplo, a percepção visual, o estado mental, a memória, a organização visual, a resposta motora, o reconhecimento do real através da sensopercepção ${ }^{19,20}$.

A revisão de literatura traz uma proposta de ilustrar como testes e avaliações psicológicas podem traduzir e auxiliar na escolha de quais pacientes com esquizofrenia podem ter o direito de dirigir automóveis ${ }^{15}$.

\section{Epidemiologia do paciente com esquizofrenia}

Em um estudo encontrou-se que quanto maior o número de internações psiquiátricas, anos de doença, e, portanto, piora do funcionamento global do paciente, menor é a chance de condução veicular ativa. Apesar desta informação, $32 \%$ dos pacientes responderam ter dirigido veículo motorizado nos últimos 12 meses. 64\% tinham licença para dirigir. Outro dado relevante encontrado foi que apenas $31,3 \%$ possuíam carro, em contraste com a população geral $(50,6 \%)$. Somado a isto, os pacientes motorizados responderam dirigir em média $4.160 \mathrm{~km} /$ ano e a população geral $14.200 \mathrm{~km} / \mathrm{ano}$, podendo mostrar que há limitação social envolvida ${ }^{18}$.

Outra pesquisa traz que $43 \%$ dos pacientes com transtorno psicóticos tinham habilitação para dirigir, além de que houve um número menor comparado com a população geral, entre aqueles que dirigiam com a carteira suspensa ${ }^{19}$.

\section{Impacto da Esquizofrenia na Direção Veicular}

No estudo de Ferreira e Simões ${ }^{15}$, observou-se, nos pacientes com esquizofrenia, maior dificuldade de controle lateral do veículo e regulação na velocidade como erros mais representativos. Verificou-se também que existem alguns estudos que sugerem um maior número de infrações às regras de trânsito e acidentes de viação em pacientes esquizofrênicos, porém esta diferença não fora observada por outros autores. Desta forma, concluise que há necessidade de estudos sistemáticos entre testes neuropsicológicos e capacidade de condução, visto que há divergências nos trabalhos.

No que diz respeito aos estudos que avaliaram funcionamento cognitivo e global, houve uma tendência de distorção de feedback visual nos pacientes em relação à população geral, além de erros de processamento sensorial. ${ }^{20}$

Por meio do clássico teste neuropsicológico ROCF (Rey-Osterrieth Complex Figure Test), constatouse que indivíduos saudáveis tiveram desempenho significativamente melhor, porém esquizofrênicos que dirigiam apresentaram resultados melhores no exame do estado mental que esquizofrênicos não motoristas. Os resultados demonstraram que o comprometimento das habilidades visuais não impede a condução das pessoas com esquizofrenia e enfatizam a importância do estado cognitivo geral para tarefas cotidianas complexas como a condução veicular. Sendo assim, reforçam a necessidade de mais estudos para conclusões e recomendam o Minimental como teste geral de cognição ${ }^{19}$.

Quando submetidos a simuladores de direção, os pacientes ultrapassaram os limites da linha branca que separa as pistas $(\mathrm{p}<0,05)$ e apresentaram maior tendência a cruzar a linha central com maior frequência que a população geral $(p=0,06)$. Ademais, pacientes apresentaram 2,5 vezes mais chance de se envolver em colisões $(p=0,09)$. Em contrapartida, os pacientes com esquizofrenia dirigiram significativamente com menor velocidade $(p<0,05)$, possivelmente como forma de compensar os possíveis prejuízos cognitivos. Levando em consideração $p$ significativamente estatístico quando $<0,05$ e que não ficou claro se um simulador de direção traz dados reais sobre habilidades humanas de condução, a pequena amostra de pacientes não permitiu chegar a conclusão apropriada tendo, assim, sugerido ao final mais estudos 22 .

Importante ressaltar que as taxas de acidente encontradas foram quase idênticas nos grupos de pacientes com transtornos psicóticos e controle, e que, além disso, taxas de acidentes com lesões podem ser equiparadas com as da população geral. Nenhum paciente com esquizofrenia apresentou fuga com seu veículo após colisão, denotando comportamento ético ${ }^{21}$. 


\section{Esquizofrenia X Outras Doenças Psiquiátricas}

Há mais expressivo impacto de outros transtornos psiquiátricos no mau comportamento do motorista, comparativamente com a Esquizofrenia. Dentre estes transtornos mentais, observa-se que os portadores de transtornos ansiosos (antigos neuróticos) e de personalidade, trazem taxas de acidente de 49-114\% maiores que na população controle. Em relação às infrações de trânsito, os pacientes com transtornos ansiosos contribuem com $135 \%$, os que possuem transtorno de personalidade com $167 \%$ a mais que o geral dos motoristas. Em contrapartida, os que possuem Esquizofrenia, trazem uma taxa de $59 \%$ a mais de infrações que o controle ${ }^{22}$.

Cabe ressaltar que pacientes com transtornos de personalidade/ansiedade, podem ser, em sua maioria, funcionais, com bom posicionamento sócioeconômico e que, do ponto de vista psicopatológico, muitas vezes passam despercebidos diante de um exame médico ou até mesmo psicológico.

No caso dos transtornos ansiosos, estes podem ser expressados em forma de crises que não estarão necessariamente presentes durante o contato com o examinador. Os transtornos de personalidade podem vir carregados de surtos, de rompantes de agressividade, raiva, comportamento explosivo, muitas vezes diante de frustrações nas relações interpessoais. São sintomas situacionais, que podem também estar ausentes no momento do exame de aptidão física e mental para a Carteira Nacional de Habilitação.

\section{Esquizofrenia X Comorbidades clínicas}

Um estudo avaliou que, das mortes na direção veicular, de 44 a $88 \%$ tem como causa a doença arterial coronariana. Na necropsia destes pacientes que faleceram dirigindo, dois terços apresentavam pelo menos uma coronária com lesão de $75 \%$. Isto reforça que a morte súbita entra como uma das principais causas de acidentes fatais no trânsito ${ }^{23}$.

Em trabalho multicêntrico realizado em quatro capitais brasileiras (Salvador, Recife, Curitiba e Brasília), nos serviços de emergência (1169 vítimas) e nos institutos médico-legais (45 vítimas fatais), observou-se que, destes pacientes que se envolveram em acidentes de trânsito, $63,5 \%$ dos homens tinham alcoolemia positiva, sendo $33,5 \%$ superior a $0,6 \mathrm{~g} / 1$ e $53,7 \%$ das mulheres apresentaram alcoolemia positiva estando 9,6\% acima do limite estabelecido pelo Código de Trânsito Brasileiro ${ }^{24}$.

Apesar de a literatura não trazer dados consistentes a respeito da prevalência de acidentes graves e fatais relacionados aos pacientes com esquizofrenia, os estudos apontam que são equiparados aos da população geral, com dimensões muito menos relevantes diante de outras morbidades, como morte súbita, doença coronariana aguda, epilepsia e uso de bebida alcóolica na direção.

Tabela 1. Levantamento dos estudos de Esquizofrenia X Direção Veicular e seus principais achados

\begin{tabular}{|c|c|c|c|c|c|}
\hline Fontes & País de origem & $\begin{array}{l}\text { Desenho do } \\
\text { estudo }\end{array}$ & Objetivos & $\mathbf{N}$ do estudo & Principais Achados \\
\hline Steinert et al., 2015 & Alemanha & Transversal & $\begin{array}{l}\text { Explora padrões de } \\
\text { mobilidade individual } \\
\text { e preditores de uso de } \\
\text { veículos motorizados } \\
\text { em uma amostra } \\
\text { representativa } \\
\text { de pessoas com } \\
\text { esquizofrenia em área } \\
\text { urbana/rural. }\end{array}$ & $\begin{array}{l}\mathrm{N}=150 \\
\text { (pacientes } \\
\text { hospitalizados } \\
\mathrm{e} \\
\text { ambulatoriais) }\end{array}$ & $\begin{array}{l}\text {-Quanto menor o } \\
\text { funcionamento social, } \\
\text { maior o } \mathrm{n}^{\circ} \text { de internações } \\
\text { e história de condução sob } \\
\text { efeito de álcool e drogas, } \\
\text { menores as chances de } \\
\text { condução ativa. } \\
\text {-Pessoas com esquizofrenia } \\
\text { não parecem representar } \\
\text { proporção relevante de } \\
\text { acidentes rodoviários. }\end{array}$ \\
\hline Palmer et al., 2000 & EUA & Transversal & $\begin{array}{l}\text { Explorar três } \\
\text { domínios do } \\
\text { status funcional } \\
\text { entre pacientes } \\
\text { esquizofrênicos } \\
\text { de meia-idade e } \\
\text { idosos que vivem } \\
\text { em comunidades } \\
\text { terapêuticas: vida } \\
\text { laboral desde o } \\
\text { início da doença, } \\
\text { independência atual } \\
\text { e status de direção } \\
\text { veicular. }\end{array}$ & $\begin{array}{l}\mathrm{N}=83 \\
\text { esquizofrênicos } \\
\mathrm{N}=46 \text { grupo } \\
\text { controle }\end{array}$ & $\begin{array}{l}\text {-Pacientes tinham menor } \\
\text { propensão a dirigir e ter } \\
\text { a carteira de habilitação; } \\
\text { tiveram pior performance } \\
\text { neuropsicológica e } \\
\text { capacidade funcional. } \\
\text {-Dentre os esquizofrênicos } \\
\text { os motoristas encontrados } \\
\text { eram predominantemente } \\
\text { do subtipo paranoide. } \\
\text {-Os sintomas negativos } \\
\text { foram ligados à maior } \\
\text { severidade em relação ao } \\
\text { status funcional. }\end{array}$ \\
\hline
\end{tabular}


Fernandes C, Esper CDA, Faiman CJS. Esquizofrenia e direção veicular: uma revisão sistemática da literatura.

continuação

\begin{tabular}{|c|c|c|c|c|c|}
\hline Fontes & País de origem & $\begin{array}{l}\text { Desenho do } \\
\text { estudo }\end{array}$ & Objetivos & N do estudo & Principais Achados \\
\hline Ferreira, et al., 2015. & Portugal & $\begin{array}{l}\text { Revisão de } \\
\text { literatura }\end{array}$ & $\begin{array}{l}\text { Documentar dados } \\
\text { de investigação } \\
\text { empírica relativos a } \\
\text { testes psicológicos } \\
\text { preditores da } \\
\text { atividade de condução } \\
\text { em pessoas com } \\
\text { doença neurológica } \\
\text { ou psiquiátrica, e } \\
\text { contribuir para a } \\
\text { sensibilização de } \\
\text { médicos sobre a } \\
\text { utilidade de uma } \\
\text { avaliação psicológica } \\
\text { diferenciada no exame } \\
\text { clínico de condutores. }\end{array}$ & 36 artigos & $\begin{array}{l}\text {-Há escassez de dados } \\
\text { empíricos sobre relação } \\
\text { entre doenças psiquiátricas } \\
\text { e capacidade de condução. } \\
\text {-Dificuldades no controle } \\
\text { das variáveis de confusão. } \\
\text {-Alguns estudos } \\
\text { sugerem um maior no de } \\
\text { infrações às regras de } \\
\text { trânsito e acidentes em } \\
\text { pacientes esquizofrênicos } \\
\text { comparados à população } \\
\text { geral, embora esta } \\
\text { diferença não tenha sido } \\
\text { observada por outros } \\
\text { autores. } \\
\text {-São necessários estudos } \\
\text { sistemáticos sobre a } \\
\text { relação entre testes } \\
\text { neuropsicológicos e a } \\
\text { capacidade de condução } \\
\text { em pessoas com doença } \\
\text { psiquiátrica. }\end{array}$ \\
\hline
\end{tabular}

\begin{tabular}{|c|c|c|c|c|c|}
\hline $\begin{array}{l}\text { Velikovsky et at., } \\
2012\end{array}$ & Israel & Transversal & $\begin{array}{l}\text { Saber se a percepção } \\
\text { visual, estado mental } \\
\text { e memória afetam a } \\
\text { aptidão para dirigir } \\
\text { em pessoas com } \\
\text { esquizofrenia da } \\
\text { mesma maneira como } \\
\text { em outras populações. }\end{array}$ & $\mathrm{N}=60$ & $\begin{array}{l}\text {-ROCF (teste } \\
\text { neuropsicológico } \\
\text { clássico) revelou que } \\
\text { habilidades dos indivíduos } \\
\text { saudáveis foi melhor } \\
\text { que dos pacientes com } \\
\text { esquizofrenia. } \\
\text {-O comprometimento das } \\
\text { habilidades visuais não } \\
\text { impedem a condução das } \\
\text { pessoas com esquizofrenia } \\
\text { e enfatiza a importância do } \\
\text { estado cognitivo geral para } \\
\text { condução. } \\
\text {-São necessários mais } \\
\text { estudos aprofundados no } \\
\text { tema. } \\
\text {-Recomenda-se teste geral } \\
\text { de cognição (Minimental) } \\
\text { para exame de aptidão de } \\
\text { condução veicular. }\end{array}$ \\
\hline Posada et al., 2006 & França & Transversal & $\begin{array}{l}\text { Avaliar o reconhecimento } \\
\text { e atribuição das ações } \\
\text { para diferenciar o real } \\
\text { do virtual, no sentido } \\
\text { de avaliar prejuízo da } \\
\text { esquizofrenia na direção } \\
\text { veicular. }\end{array}$ & $\mathrm{N}=30$ & $\begin{array}{l}\text {-Houve uma tendência dos } \\
\text { pacientes esquizofrênicos } \\
\text { em realizar erro de janela } \\
\text { do estágio sensorial do } \\
\text { processamento, denotando } \\
\text { possível prejuízo cognitivo } \\
\text { visual. } \\
\text {-Estudos complementares } \\
\text { são necessários. }\end{array}$ \\
\hline
\end{tabular}

continua 


\begin{tabular}{|c|c|c|c|c|c|}
\hline Fontes & País de origem & $\begin{array}{l}\text { Desenho do } \\
\text { estudo }\end{array}$ & Objetivos & N do estudo & Principais Achados \\
\hline Germain, et al., 2005 & EUA & Caso-controle & $\begin{array}{l}\text { Responder sobre } \\
\text { competência da direção } \\
\text { na esquizofrenia, } \\
\text { visto que maioria } \\
\text { da população com } \\
\text { esquizofrenia vive e } \\
\text { possivelmente dirige na } \\
\text { comunidade. }\end{array}$ & $\begin{array}{l}\mathrm{N}=12 \\
\text { esquizofrênicos } \\
\mathrm{N}=25 \text { grupo } \\
\text { controle }\end{array}$ & $\begin{array}{l}\text {-Apesar da amostra ser } \\
\text { pequena e não conseguir } \\
\text { trazer dados reais, os } \\
\text { pacientes ultrapassaram } \\
\text { limites da linha branca da } \\
\text { pista mais que população } \\
\text { controle ( }<<0,05 \text { ) e } \\
\text { tenderam a cruzar a } \\
\text { linha central com maior } \\
\text { frequência ( } p=0,06 \text { ). } \\
\text {-Pacientes apresentaram } \\
2,5 \text { vezes mais chance de } \\
\text { se envolver em colisões } \\
\text { (p=0,09). } \\
\text {-Indivíduos com } \\
\text { esquizofrenia dirigiram } \\
\text { com menor velocidade } \\
(p<0,05 \text { ), provavelmente } \\
\text { para compensar prejuízos } \\
\text { cognitivos. } \\
\text { - Mais estudos } \\
\text { complementares são } \\
\text { necessários na área. }\end{array}$ \\
\hline Crancer, et al., 1969 & EUA & Transversal & $\begin{array}{l}\text { O objetivo do estudo } \\
\text { foi determinar se há } \\
\text { alguma correlação } \\
\text { entre alguns distúrbios } \\
\text { mentais e performance } \\
\text { na condução veicular, } \\
\text { e analisar a natureza } \\
\text { da correlação, uma } \\
\text { vez encontrada. }\end{array}$ & $\begin{array}{l}\mathrm{N}=271 \\
\text { pacientes } \\
\text { psiquiátricos, } \\
\text { sendo } 97 \mathrm{com} \\
\text { transtorno } \\
\text { psicótico }\end{array}$ & $\begin{array}{l}\text {-Dos pacientes com t. } \\
\text { psicótico, } 43 \% \text { tinham } \\
\text { habilitação para dirigir. } \\
\text {-Taxas de acidente quase } \\
\text { idênticas nos grupos de } \\
\text { transtornos psicóticos e } \\
\text { população controle. } \\
\text {-Taxa de infrações pouco } \\
\text { maiores nos pacientes com } \\
\text { transtornos psicóticos que } \\
\text { na população geral. } \\
\text {-Os acidentes com lesões } \\
\text { foram equiparados aos da } \\
\text { população geral. } \\
\text {-Mais estudos são } \\
\text { necessários. }\end{array}$ \\
\hline
\end{tabular}

\section{DISCUSSÃO}

Os pacientes trazem quadros clínicos extremamente heterogêneos, somados às suas comorbidades, o que exerce distinta influência no funcionamento cognitivo e psicomotor, acarretando diferentes possibilidades no comportamento da condução.

Além da complexidade per si da Esquizofrenia em seu espectro sintomático, vale relembrar o impacto dos medicamentos psicotrópicos e o uso associado de álcool e drogas ilícitas, muito comumente encontrado nos transtornos mentais em geral.

Pensando na mobilidade como importante papel na saúde, bem-estar e participação social, seria de extrema importância a realização de mais estudos aprofundados neste tema, sistematizados, com maior amostra populacional e mais testes empregados.
No cenário prático do exame de aptidão física e mental, em muitas situações ocorre como um "carimbo" identificando "esquizofrênico" e já de imediato este indivíduo é taxado como inapto. Neste sentido, são necessárias melhorias na estratificação da gravidade do transtorno psicótico e de seu impacto neuropsicomotor para direção veicular.

Existe uma falsa ideia de que todo paciente com esquizofrenia seria criminalmente inimputável perante a lei, e tal fato encontrar-se-ia em desacordo com a lei $\mathrm{n}^{\circ}$ 9.503, artigo 140 do Código de Trânsito Brasileiro, em que se diz que o candidato deve ser penalmente imputável para ter o direito de condução veicular ${ }^{25}$.

Desta maneira, denota-se necessidade de exame e investigação clínica mais apuradas no momento do exame físico e mental deste candidato em questão. Para isso, coloca-se, como sugestão: 
1) $\mathrm{O}$ paciente que declarar ser portador de esquizofrenia no questionário de habilitação/ renovação da licença veicular, deverá apresentar exames complementares com laudo e parecer favorável de especialista, no caso, psiquiatra que o assiste em seu tratamento;

2) Poderia ser confeccionado relatório padronizado de preenchimento do psiquiatra, com questões relevantes como idade de início da doença, número de surtos, internações prévias, presença de sintomas negativos, uso de psicotrópicos, exame do estado mental, comprometimento cognitivo e social;

3) Sugere-se a realização de testes neuropsicológicos para os pacientes com transtornos psicóticos, a fim de estimar comprometimento psíquico;

4) Seria de suma importância, assim como nos casos de deficiência física, a realização de junta médica técnica, acompanhando estes pacientes em situações práticas de direção veicular, para dimensionamento de suas capacidades em cenários diversos.

5) Em última instância, em casos de estar apto para a direção veicular, poderia ser diminuída a validade da carteira nacional de habilitação para prazo de um ano, por exemplo, a fim de avaliações periódicas e assim estabelecer ideia de evolução e progressão da doença.

6) Existem testes simuladores de direção veicular que poderiam ser utilizados nestes casos como instrumento complementar na avaliação cognitiva.

Em suma, torna-se de extrema importância investigação mais apurada destes pacientes, para que não sejam indevidamente impedidos de exercer o ato da direção veicular. À medida que mais estudos surgirem, os profissionais da Medicina de Tráfego obterão mais embasamento teórico em sua avaliação mental.

\section{CONCLUSÃO}

Diante dos dados expostos, fica evidente a relação entre Esquizofrenia e prejuízo cognitivo. Porém os estudos traçados não permitiram exercer com solidez a aferição destas perdas mentais, e tampouco demonstrar o impacto na direção veicular. Em suma, torna-se de extrema importância a investigação mais apurada destes pacientes, para que não sejam indevidamente impedidos de exercer o ato da direção veicular. À medida que mais estudos surgirem, os profissionais da Medicina de Tráfego obterão mais embasamento teórico em sua avaliação mental.

Fernandes C, Esper CDA, Faiman CJS. Schizophrenia and vehicle driving: a systematic literature review. Saúde, Ética \& Justiça. 2017;22(2):72-80.

\begin{abstract}
Introduction: The diagnosis of schizophrenia has, over the years, been charged with a historical process of transformation under the eyes of society. In this context, schizophrenia is seen as a degenerative disease, with progressive dementia, with global impairment of the patient's functions and cognitive decline, generating, very commonly, a life marked by prejudice and exclusion, including in the area of mobility with respect to vehicle steering. Concomitant to these data, there is a shortage of research work on car accidents in drivers with schizophrenia, although some studies point to a lack of a higher potential risk factor. Objective: The objective of this literature review for studies about the literature that bring information about the patient with schizophrenia and vehicular direction, seeking respondents may or may not be allowed to drive cars through mental / psychological examination. Methodology: A systematic review of the scientific literature on a relationship between schizophrenia and vehicular direction was carried out. Results: There are studies that suggest a greater number of infractions to the rules of traffic and traffic accidents in schizophrenic patients, but these data are not observed by other authors. There was a tendency for distortion of visual feedback in patients in relation to the general population, in addition to sensory processing errors. Schizophrenics who drove presented themselves better at mental state examination than schizophrenic non-drivers. The accident rates found were very similar in the groups of patients with psychotic disorders and control, and that in addition, injury rates can be equated with the general population. Conclusion: Given the data presented, evidence a relationship between schizophrenia and cognitive impairment. However, the studies traced can not be applied with solidness to the measurement of these mandates, nor they demonstrate the impact in the vehicular sense. In short, these patients become extremely important in the investigation, so that they are not unduly prevented from exercising the act of the vehicular direction. As more studies emerge, Traffic Medicine practitioners gain more theoretical grounding in their mental assessment.
\end{abstract}

KEY WORDS: Schizophrenia; Automobile Driving.

\title{
REFERÊNCIAS
}

1. Silva RCB. Esquizofrenia: Uma revisão. Psicologia USP [Internet]. 2006 [acesso em 2016 ago. 01]; 17(4):263-85. Disponível em: http://www.scielo.br/pdf/pusp/v17n4/ v17n4a14
2. Souza FGM, Abreu GC, Nascimento JR, Leite RTP, Oliveira RLJ. DSM 5: O que realmente mudou?. In: Associação Brasileira de Psiquiatria, Nardi AE, Silva AG, Quevedo JL, organizadores. PROPSIQ Programa de Atualização em 
Psiquiatria: Ciclo 5. Porto Alegre: Artmed Panamericana; 2015. p. 9-49.

3. American Psychiatric Association. Manual diagnóstico e estatístico de transtornos mentais - DSM-5. $5^{\mathrm{a}}$ ed. Porto Alegre: Artmed; 2014

4. Cooper JE, Bostock J. Relationship between schizophrenia, social disability, symptoms and diagnosis. In: Henderson, Burrows, editors. Handbook of Social Psychiatry. London: Elsevier Science Publishers; 1988. p. 317-30.

5. Chaves AC. Diferenças entre os sexos na esquizofrenia Rev Bras Psiquiatr [Internet]. 2000 [acesso em 2016 ago. 01]. 22(Supl I):21-2. Disponível em: http://www.scielo.br/ $\mathrm{pdf} / \mathrm{rbp} / \mathrm{v} 22 \mathrm{~s} 1 / \mathrm{a} 08 \mathrm{v} 22 \mathrm{~s} 1 . \mathrm{pdf}$

6. Rizzo M, Kellison IL. The brain on the road. In: Marcotte TD, Grant I, editors. Neuropsychology of everyday functioning. New York: Guilford Press; 2010. p. 168-207.

7. Marcotte TD, Scott JC. Neuropsychological performance and the assessment of driving behaviour. In: Grant I, Adams K, editors. Neuropsychological assessment of neuropsychiatric and neuromedical disorders. New Yorl: Oxford University Press; 2009. p. 652-87.

8. Wolfe PL, Clark JÁ. Driving capacity. Civil capacities in clinical neuropsychology: Research findings and practical applications. New York: Oxford University Press; 2012. p. 121-38.

9. Dassanayake T, Michie P, Carter G, Jones A. Effects of benzodiazepines, antidepressants and opioids on driving: A systematic review and meta-analysis of epidemiological and experimental evidence. Drug Saf. 2011;34(2):125-56. DOI: http://dx.doi.org/10.2165/11539050-000000000-00000

10. Hambrecht M, Lammertink M, Klosterkötter J, Matuschek E, Pukrop R. Subjective and objective neuropsychological abnormalities in a psychosis prodrome clinic. Br J Psychiatry Suppl. 2002;43:s30-7.

11. Harris M. Psychiatric conditions with relevance to fitnes to drive. Adv Psychiatr Treat. 2000;6(4):261-9. DOI: https://doi.org/10.1192/apt.6.4.261

12. Silverstone T. The influence of psychiatric disease and its treatment on driving performance. Int Clin Psychopharmacol. 1988;3(suppl 1):59-66.

13. Lundqvist A. Neuropsychological aspects of driving characteristics. Brain Inj. 2001;15:981-94.

14. Adura FE. Manual do Exame de Aptidão Física e Mental para Condutores e Candidatos a Condutores de Veículos
Automotores. São Paulo: ABRAMET, 2013.

15. Ferreira IS, Simões MR. Contributo da avaliação psicológica no exame clínico de condutores com doença neurológica e psiquiátrica: revisão teórica. Rev Port Saúde Pública. 2015;33(1):57-70. DOI: https://doi.org/10.1016/j. rpsp.2014.03.003

16. Palmer BW, Heaton RK, Gladsjo JÁ, Evans JD, Patterson TL, Golshan S, et al. Heterogeneity in functional status among older outpatients with schozophrenia: employment history, living situation and driving. Schizophr Res. 2002;55(3):205-15.

17. Leung J, Deane FP, Taylor JE, Bliokas VV. Anxiety in driving assessment of individuals with cognitive impairment. Disabil Rehabil. 2009;31(20):1700-8. DOI: http://dx.doi.org/10.1080/09638280902738581

18. Steinert T, Veit F, Schmid P, Jacob Snellgrove B, Borbé R. Participating in mobility: People with schizophrenia driving motorized vehicles. Psychiatry Res. 2015;228(3):719-23. DOI: http://dx.doi.org/10.1016/j.psychres.2015.05.034

19. Lipskaya-Velikovsky L, Kotler M, Weiss P, Kaspi M, Gamzo S, Ratzon N. Car driving in schizophrenia: can visual memory and organization make a difference?, Disabil Rehabil. 2013;35(20):1734-9. DOI: http://dx.doi.org/10.31 09/09638288.2012.753116

20. Posada A, Franck N, Augier S, Georgieff N, Jeannerod M. Altered processing of sensorimotor feedback in schizophrenia. C.R. Biol. 2007;330(5):382-8. DOI: http:// dx.doi.org/10.1016/j.crvi.2007.02.003

21. St Germain SA, Kurtz MM, Pealrson GD, Astur RS. Driving simulator performance in Schizophrenia. Schizophr Res. 2005;74(1):121-2.

22. Crancer A, Quiring DL. The Mentally Ill as Motor Vehicle Operators. Am J Psychiatry. 1969;126(6):807-13. DOI: http://dx.doi.org/10.1176/ajp.126.6.807

23. Christian MS. Incidence and implications of natural death of road users. Br Med J. 1988;297(6655):1021-4.

24. Nery AF, Medina MG, Melcope AG, Oliveira EM. Impacto do uso de álcool e outras drogas em vítimas de acidentes de trânsito. Brasília: ABDETRAN; 1997.

25. Brasil. Presidência da República, Casa Civil, Subchefia para Assuntos Jurídicos. Lei No 9.503, de 23 de Setembro de 1997. Institui o Código de trânsito Brasileiro [Internet]. Brasília, DF; 1997. [acesso em 2016 ago. 01]. Disponível em: http://www.planalto.gov.br/ccivil_03/leis/L9503.htm 